\title{
Specific labelling by peanut agglutinin of the outer acrosomal membrane of the human spermatozoon
}

\author{
D. Mortimer, E. F. Curtis and R. G. Miller* \\ Endocrine/Infertility Clinic, Department of Obstetrics \& Gynaecology, and *Department of \\ Anatomy, University of Calgary, Health Sciences Centre, 3330 Hospital Drive N.W., Calgary, \\ Alberta, Canada $T 2 N 4 N 1$
}

\begin{abstract}
Summary. Experiments to bind fluorescein-conjugated Arachis hypogea (peanut) agglutinin (FITC-PNA) to washed human spermatozoa demonstrated that this lectin binds to the acrosome region in air-dried preparations. Since there was no binding when labelling was performed in suspension, and comparable labelling to that seen in air-dried preparations was seen when spermatozoa treated with saponin (to lyse the plasma membrane) were labelled in suspension, the lectin must bind to an intracellular structure, probably the outer acrosomal membrane. This was confirmed by ultrastructural localization of colloidal gold-conjugated lectin in saponin-treated spermatozoa. Treatment of spermatozoa with the detergent Nonidet P-40 caused a marked change in the binding pattern: more spermatozoa showed binding in the equatorial segment of the acrosome with no binding in the anterior cap region. A comparable, less marked, change was seen when spermatozoa were incubated overnight under conditions known to support the capacitation and spontaneous acrosome reactions. Treatment with the calcium ionophore A23187 for $1 \mathrm{~h}$ to induce acrosome reactions artificially in uncapacitated spermatozoa resulted in the appearance of patchy acrosome fluorescence. From these experiments it is concluded that PNA binds specifically to the outer acrosomal membrane, and that FITC-PNA-labelling may be used to monitor the human sperm acrosome reaction.
\end{abstract}

\section{Introduction}

During the past 20 years there have been numerous studies on the use of lectins as probes of the mammalian sperm surface (reviewed by Koehler, 1981). These studies have provided valuable information on the localized specializations of this highly differentiated cell membrane. Of particular interest has been the documentation of surface phenomena associated with capacitation and the acrosome reaction. Fluorescein-conjugated Ricinus communis agglutin-60 (RCA-60) has been used to monitor the acrosome reaction of human spermatozoa (Talbot \& Chacon, 1980, 1981), although its use has been rather limited due to the high toxicity of ricin.

Our preliminary observations on the surface mapping of human spermatozoa with fluorescent lectins were aimed at identifying a possible substitute for RCA-60 as an acrosomal probe. In this respect Arachis hypogea (peanut) agglutinin (PNA) was found to be of particular interest, and has also been the subject of several recent publications from other laboratories (Kallajoki et al., 1985; Lee \& Damjanov, 1985).

The experiments reported in this paper describe the identification and initial evaluation of PNA as a marker for the acrosomal region of human spermatozoa, including the ultrastructural localization of its binding to the outer acrosomal membrane. 


\section{Materials and Methods}

Materials. Semen samples were obtained from normal healthy donors by masturbation at the laboratory. Only samples with completely normal semen characteristics were used in these studies (Mortimer, 1985a). Aliquants of liquefied semen were used at $30-60 \mathrm{~min}$ after ejaculation and sperm suspensions were prepared by using Percoll gradients (Dravland \& Mortimer, 1985) or a "wash and swim-up" procedure (Mortimer, 1985b). Sperm suspensions were used at approximately $5 \times 10^{6} / \mathrm{ml}$ for making air-dried preparations and concentrations of $20-25 \times 10^{6} / \mathrm{ml}$ for labelling in suspension. All centrifugations of prepared sperm suspensions were at $600 \mathrm{~g}$ for $6 \mathrm{~min}$.

Various media and buffers were used in the experiments: a modified Tyrode's medium ('MT medium', compo-

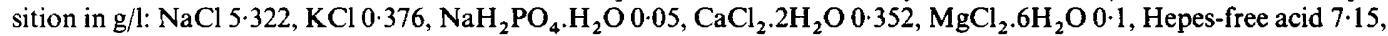
$\mathrm{NaHCO}_{3} 2 \cdot 1$, human serum albumin (Cohn fraction $\mathrm{V}$ ) $12 \cdot 0$, glucose $0 \cdot 9$, sodium pyruvate $0 \cdot 0276$ and sodium lactate, $60 \%$ syrup, $1.868 \mathrm{ml} / \mathrm{l}$; final osmolarity $295-300 \mathrm{mosmol}$ and $\mathrm{pH} 7.65$ after adjustment with $10 \mathrm{~N}-\mathrm{NaOH}$ : Dravland \& Mortimer, 1985); phosphate-buffered saline (PBS, composition in $\mathrm{g} / \mathrm{l}: \mathrm{NaCl} 8 \cdot 0, \mathrm{CaCl}_{2} .2 \mathrm{H}_{2} \mathrm{O} 0 \cdot 132, \mathrm{KCl} 0 \cdot 2$, $\mathrm{KH}_{2} \mathrm{PO}_{4} 0 \cdot 2, \mathrm{MgSO}_{4} 0 \cdot 059, \mathrm{Na}_{2} \mathrm{HPO} 1 \cdot 15 ; 302 \mathrm{mosmol}, \mathrm{pH} 7 \cdot 35$ ); and Maunsbach's phosphate buffer (MPB, composition in g/l: $\mathrm{NaH}_{2} \mathrm{PO}_{4} \cdot \mathrm{H}_{2} \mathrm{O} 2 \cdot 98, \mathrm{Na}_{2} \mathrm{HPO}_{4} \cdot 7 \mathrm{H}_{2} \mathrm{O} 30 \cdot 4$ : final molarity $135 \mathrm{mM}, 298 \mathrm{mosmol}$, pH 7.35: Glauert, 1975).

Conjugated preparations of peanut agglutinin with fluorescein isothiocyanate (FITC) and colloidal gold (10 nm diameter particles) were purchased from EY Laboratories (San Mateo, CA, U.S.A.). All slides of FITC-PNAlabelled spermatozoa were mounted with $22 \times 22 \mathrm{~mm}$ coverslips using a PBS:glycerol mixture $(1: 1, \mathrm{v} / \mathrm{v})$ and examined using a Leitz Orthoplan microscope equipped with Ploemopak epi-illumination module (I.2 filter cube) and mercury vapour u.v. source.

Experiment 1: qualitative evaluation of FITC-PNA labelling. Spermatozoa recovered from Percoll gradients in MT medium were labelled in suspension or after air drying. Duplicate samples were fixed in suspension for $3 \mathrm{~h}$ with $3.0 \%$ (v/v) glutaraldehyde in $100 \mathrm{~mm}$-cacodylate buffer $\mathrm{pH} 7.5$ before labelling on slides. Fixed spermatozoa were washed with cacodylate buffer containing $100 \mathrm{~mm}$-glycine to react with any remaining aldehyde and then again with fresh cacodylate buffer before use.

Labelling in suspension used FITC-PNA at a final concentration of $10 \mu \mathrm{g} / \mathrm{ml}$ for $15 \mathrm{~min}$, followed by addition of an excess volume of medium and centrifugation. After discarding the supernatant the spermatozoa were resuspended to the original aliquant volume before being transferred to alcohol-cleaned slides and allowed to air dry.

Air-dried preparations were made by spreading $10 \mu \mathrm{l}$ sperm suspension over about $1 \mathrm{~cm}^{2}$ using a pipette tip and leaving at ambient temperature. Labelling was accomplished using $20 \mu \mathrm{l}$ of $100 \mu \mathrm{g}$ FITC-PNA/ml for $10 \mathrm{~min}$ in a humid chamber at $+4^{\circ} \mathrm{C}$. Slides were then rinsed gently with PBS and mounted using PBS:glycerol.

Fixed spermatozoa were labelled on slides using an equal volume of $1 \mathrm{mg}$ FITC-PNA/ml for $10 \mathrm{~min}$ in a humid chamber at $+4^{\circ} \mathrm{C}$. These slides were then treated as described for air-dried preparations. Microscopic examination revealed four patterns of fluorescence (Fig. 1) and these categories were used in scoring subsequent experiments.

Experiment 2: Nonidet P-40 permeabilization of spermatozoa. Following the report of Kallajoki et al. (1985) the detergent Nonidet P-40 (NP-40: Sigma Chemical Co., St Louis, MO, U.S.A.) was used to permeabilize the sperm plasma membrane to reveal intracellular binding sites.

Percoll gradient-prepared sperm suspensions were adjusted to about $25 \times 10^{6} / \mathrm{ml}$ in MT medium. Air-dried and suspension-labelled controls were prepared as described for Exp. 1. Duplicate samples of the sperm suspension were treated with an excess volume of $0.1 \%(\mathrm{v} / \mathrm{v})$ NP-40 in MPB for $15 \mathrm{~min}$ at ambient temperature. An equal volume of MT medium was then added, the tubes were centrifuged and the supernatants discarded. The spermatozoa were resuspended to the original aliquant volumes and used to make air-dried and suspension-labelled FITC-PNA preparations as described above.

Duplicate preparations were made for each treatment group in two separate experiments. Unlabelled cells were identified by brief examination under phase-contrast illumination. Normally 300 spermatozoa were counted per slide and their fluorescence was scored according to the patterns described in Fig. 1.

Experiment 3: saponin permeabilization of spermatozoa. In this experiment the detergent saponin (Fisher Chemical Co., Fair Lawn, NJ, U.S.A.) was used to lyse the sperm plasma membrane. Spermatozoa were prepared by swim-up migration and adjusted to about $25 \times 10^{6} / \mathrm{ml}$. Aliquants of sperm suspension were treated with 9 volumes of $0 \cdot 1 \%$ $(w / v)$ saponin in PBS or with PBS alone at ambient temperature for $5 \mathrm{~min}$. Spermatozoa were labelled with FITCPNA after air drying or in suspension and scored under epifluorescence as described for Exp. 2.

Experiment 4: changes in PNA labelling related to acrosome reactions. Eight donor sperm suspensions recovered from Percoll gradients were prepared in MT medium at $25 \times 10^{6}$ spermatozoa/ml. Air-dried FITC-PNA-labelled preparations were made as described above. The remaining sperm suspensions were adjusted to $5 \times 10^{6} / \mathrm{ml}$, gassed with $5 \% \mathrm{CO}_{2}$ in air, and incubated overnight $(20 \mathrm{~h})$ at $37^{\circ} \mathrm{C}$. After this capacitation incubation, aliquants of the sperm suspensions were used to make air-dried preparations for PNA labelling.

Another 5 donor sperm suspensions recovered from Percoll gradients were incubated at ambient temperature for $60 \mathrm{~min}$ in MT medium with and without the calcium ionophore A23187 (1:1 Ca-Mg salt: Sigma) at a final concentration of $50 \mu \mathrm{M}$ to induce acrosome reactions in uncapacitated spermatozoa (Jamil \& White, 1981). Air-dried FITC-PNA-labelled preparations were made from the control and treated sperm suspensions.

Usually, 3 preparations were scored for each sample (500 spermatozoa/slide) according to the categories of fluorescence described in Fig. 1. However, in the A23187-treated spermatozoa a large proportion of the cells showed a 


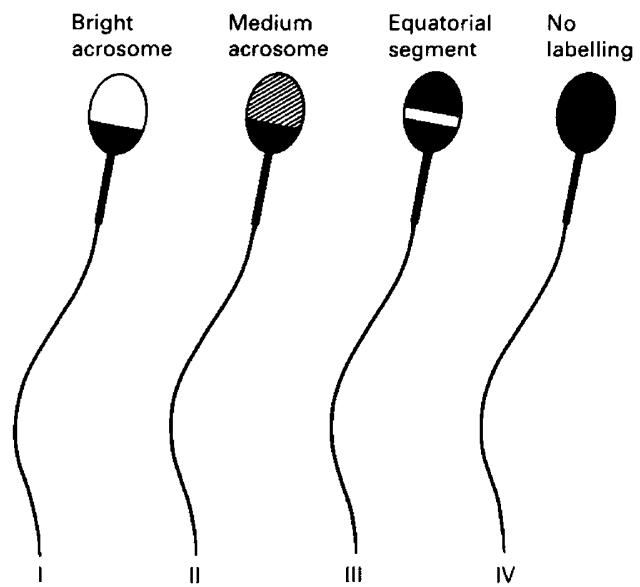

Fig. 1. Patterns of fluorescence seen after labelling of air-dried human spermatozoa with FITCPNA lectin.

patchy fluorescence over the acrosomal cap. Consequently, a fifth category (type IIa), intermediate between patterns II and III, had to be created. Occasionally one of the three slides did not show good labelling. Therefore, when the counts showed $>10 \%$ difference between the major class frequencies (i.e. classes with $>20 \%$ of the spermatozoa) in one of the slides compared to the other two then this slide was discarded. Results were the mean values obtained from the groups of 2 or 3 slides scored at each time point.

Experiment 5: ultrastructural localization of $P N A$ labelling. Saponin-permeabilized spermatozoa were prepared as described for Exp. 3 and incubated with $70 \mu \mathrm{g}$ colloidal gold conjugated with PNA/ml for $15 \mathrm{~min}$ at ambient temperature. After washing with a large excess of MT medium the spermatozoa were fixed using $4.0 \%(\mathrm{v} / \mathrm{v})$ glutaraldehyde in $200 \mathrm{~mm}$-cacodylate buffer with $4.0 \%(\mathrm{w} / \mathrm{v})$ sucrose for $60 \mathrm{~min}$ at ambient temperature. Post-fixation and pellet encapsulation using bovine serum albumin were performed as described by Stevens \& Boadle (1987). After dehydration in a graded series of acetones, pieces of pellet were embedded in an araldite/epon mixture using propylene oxide as the transition solvent.

Silver-gold sections were cut on a Reichert Om-U3 ultramicrotome with a diamond knife and viewed without further staining in a Philips EM400 operating at $80 \mathrm{kV}$.

Experiment 6: formaldehyde fixation of PNA-labelled spermatozoa. In this experiment spermatozoa were recovered from Percoll gradients, adjusted to $25 \times 10^{6} / \mathrm{ml}$, and air-dried preparations were made as described above. One set of preparations was labelled with FITC-PNA and scored immediately (Group A, Day 1). Others were labelled with PNA and then fixed with formaldehyde (Group B: see below), or stored in a dry, dark container and then labelled with PNA and scored on subsequent days (Group A, Days 2, 3 etc.). Formaldehyde was used at $3.5 \%$ (w/v paraformaldehyde) in MPB and slides were fixed by immersion in Coplin-type staining jars of fixative for 15 min at ambient temperature. Slides were then rinsed gently with fresh MPB and allowed to air dry. Fixation and air-drying procedures were carried out in the dark to prevent inactivation of the FITC. One set of these PNA-labelled-and-fixed preparations was scored immediately (Day 1), the others were stored in a dry, dark container and scored later. Usually, 3 slides were scored for each preparation method at each time point as described for Exp. 4. The results presented are the mean values for each set of slides scored.

\section{Results}

\section{Qualitative evaluation of lectin labelling of human spermatozoa}

Rather different patterns of fluorescence were seen depending whether PNA labelling was performed in suspension or on air-dried smears (see Tables 1 and 2; Figs 2-4). When spermatozoa fixed in suspension with glutaraldehyde were labelled with PNA after air drying, comparable patterns of fluorescence to the labelling in suspension of unfixed cells were found. These preparations demonstrated that PNA bound specifically to the acrosome region of the spermatozoon. 

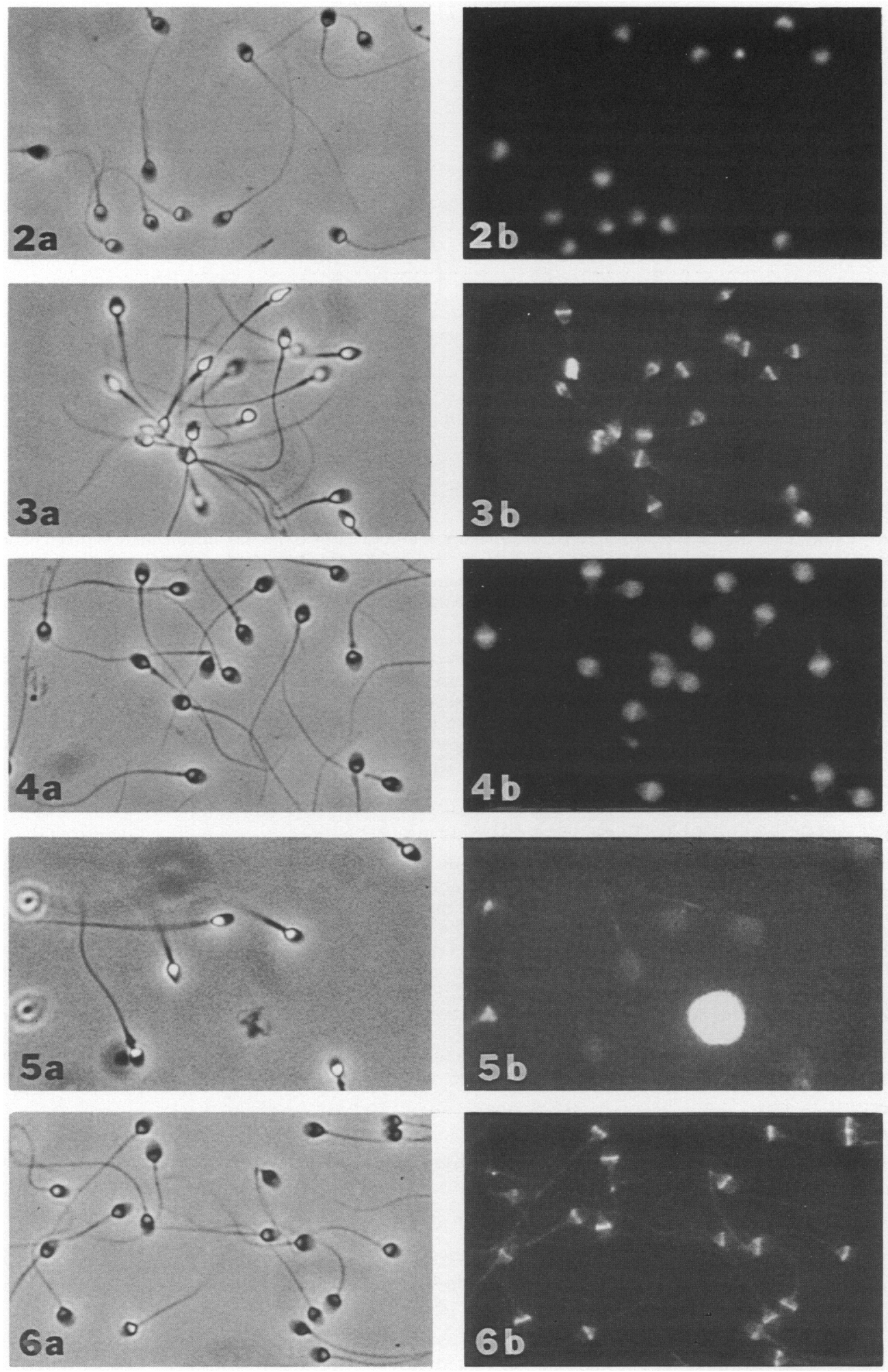
Table 1. Percentages of spermatozoa in each of the 4 classes of PNA-

labelling (see Fig. 1) in Exp. 2

\begin{tabular}{|c|c|c|c|c|c|c|c|c|}
\hline \multirow[b]{3}{*}{ Group } & \multicolumn{8}{|c|}{ Pattern of fluorescence ( $\%$ of spermatozoa) } \\
\hline & \multicolumn{4}{|c|}{ Replicate 1} & \multicolumn{4}{|c|}{ Replicate 2} \\
\hline & I & II & III & IV & $\mathbf{I}$ & II & III & IV \\
\hline$A^{*}$ & 76.0 & $8 \cdot 0$ & 3.8 & $12 \cdot 2$ & $82 \cdot 4$ & $7 \cdot 4$ & 1.5 & 8.7 \\
\hline $\mathrm{B}+$ & 0.5 & 5.0 & 4.8 & $89 \cdot 7$ & $1 \cdot 2$ & 1.6 & $1 \cdot 2$ & 96.0 \\
\hline $\mathrm{C}_{\ddagger}^{\ddagger}$ & 0.0 & $3 \cdot 1$ & $57 \cdot 1$ & $39 \cdot 8$ & $0 \cdot 1$ & $4 \cdot 3$ & 88.6 & $7 \cdot 0$ \\
\hline$D \S$ & $0 \cdot 5$ & $7 \cdot 2$ & 48.6 & $43 \cdot 7$ & 0.5 & $10 \cdot 9$ & $86 \cdot 6$ & $2 \cdot 0$ \\
\hline
\end{tabular}

*Air-dried control preparations.

†Suspension-labelled control preparations.

†Spermatozoa labelled in air-dried preparations.

$\S$ Spermatozoa labelled in suspensions after Nonidet P-40 treatment.

Table 2. Percentages of spermatozoa in each class of PNAlabelling (see Fig. 1) after labelling of a sperm sample in airdried preparations or in suspension after treatment with $0.0 \%$ or $0.1 \%$ saponin in PBS

\begin{tabular}{lcrrrr}
\hline & & \multicolumn{4}{c}{$\begin{array}{c}\text { Pattern of fluorescence } \\
\text { (\% of spermatozoa) }\end{array}$} \\
\cline { 3 - 6 } Saponin & PNA-labelling & \multicolumn{1}{c}{ I } & II & III & IV \\
\hline $0.0 \%$ & Suspension & 0.0 & $6 \cdot 2$ & $0 \cdot 8$ & $93 \cdot 0$ \\
& Air-dried & $32 \cdot 3$ & $44 \cdot 5$ & $2 \cdot 0$ & $21 \cdot 2$ \\
$0.01 \%$ & Suspension & 25.5 & $25 \cdot 1$ & $3 \cdot 2$ & $46 \cdot 2$ \\
& Air-dried & 20.8 & $43 \cdot 0$ & $3 \cdot 2$ & $33 \cdot 1$ \\
\hline
\end{tabular}

This binding was inhibited by the presence of $200 \mathrm{~mm}$ of the hapten sugar $\beta$-D-galactose (Fig. 5). However, since binding was seen only in air-dried preparations the PNA was obviously bound to an intracellular structure. To allow labelling with gold-PNA in suspension it was necessary to evaluate permeabilization procedures before studying the ultrastructural localization of the PNA labelling.

\section{Labelling of permeabilized spermatozoa with PNA}

Kallajoki et al. (1985) reported that PNA bound heavily to the acrosomal region of human spermatozoa, and that a similar binding pattern was obtained with cells permeabilized using the detergent Nonidet P-40. However, when we attempted to repeat this (Exp. 2) we found that, irrespective of whether NP-40-treated spermatozoa were labelled with PNA in suspension or after airdrying, the patterns of binding were consistently different from those of untreated spermatozoa (Table 1). While the control sperm preparations showed the pattern of binding expected from Exp.

Figs 2-6. Matched phase contrast (a) and fluorescence (b) micrographs of FITC-PNA-labelled human spermatozoa after air-drying (Fig. 2); after treatment with saponin and labelled in suspension (Fig. 3), after air-drying (Fig. 4), or in suspension but in the presence of $200 \mathrm{~mm}-\beta$ D-galactose (Fig. 5); and after treatment with NP-40 and air-drying (Fig. 6). See text for descriptions. $\times 650$. 
Table 3. Percentages of spermatozoa in each class of PNA-labelling (see Fig. 1) before and after $20 \mathrm{~h}$ of capacitation incubation

\begin{tabular}{lrrrc}
\hline & \multicolumn{4}{c}{ Pattern of fluorescence (\% of spermatozoa) } \\
\cline { 2 - 5 } Preincubation & \multicolumn{1}{c}{ I } & II & III & IV \\
\hline $0 \mathrm{~h}$ & $76.4 \pm 6.7$ & $11 \cdot 2 \pm 3 \cdot 8$ & $9.4 \pm 3 \cdot 1$ & $2.9 \pm 0.5$ \\
$20 \mathrm{~h}$ & $63.9 \pm 7 \cdot 1^{*}$ & $9.8 \pm 3 \cdot 3$ & $15 \cdot 5 \pm 5 \cdot 4 \dagger$ & $10 \cdot 9 \pm 1.9^{* *}$ \\
\hline
\end{tabular}

Values are mean \pm s.e.m. of 8 experiments. Superscripts denote significant differences within fluorescence groups as determined by paired $t$ tests: ${ }^{*} P<0.01,{ }^{* *} P<0.001,+0.05<P<0.1$.

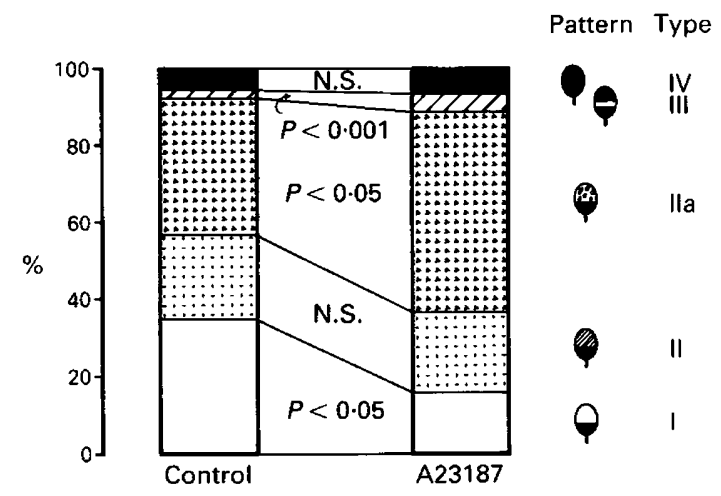

Fig. 7. Percentages of human spermatozoa showing the various patterns of FITC-PNA labelling (see Fig. 1 and text) before and after treatment with A23187 for $60 \mathrm{~min}$. Values are means of 5 experiments.

1, NP-40-treated spermatozoa showed a preponderance of only equatorial segment fluorescence (Fig. 6).

Saponin-treated spermatozoa, whether labelled with PNA in suspension or in air-dried preparations (Exp. 3), showed patterns of fluorescence similar to those of untreated spermatozoa labelled in air-dried smears (Table 2; Figs 3 and 4). Saponin-treated spermatozoa labelled in suspension showed more unlabelled cells than did air-dried controls, but less than suspension-labelled controls, indicating that many spermatozoa had been permeabilized. Air drying resulted in a shift towards category II, suggesting an increased fragility of the outer acrosomal membrane after saponin treatment.

\section{Changes in PNA labelling related to acrosome reactions}

In 8 donor sperm populations (Exp. 4, Table 3) the proportion of the spermatozoa showing strong PNA labelling of the acrosome region (Type I fluorescence) was reduced after a 20 -h overnight incubation under conditions known to support in-vitro capacitation and the acrosome reaction (Mortimer, 1986). This difference was statistically significant $(P<0.01$, paired $t$ test). Concomitant with this change were increases in the proportions of Type III spermatozoa (labelled only in the equatorial segment of the acrosome, $P<0.01$ ) as well as unlabelled, presumably acrosome-less (Type IV) spermatozoa $(0 \cdot 05<P<0 \cdot 1)$. 

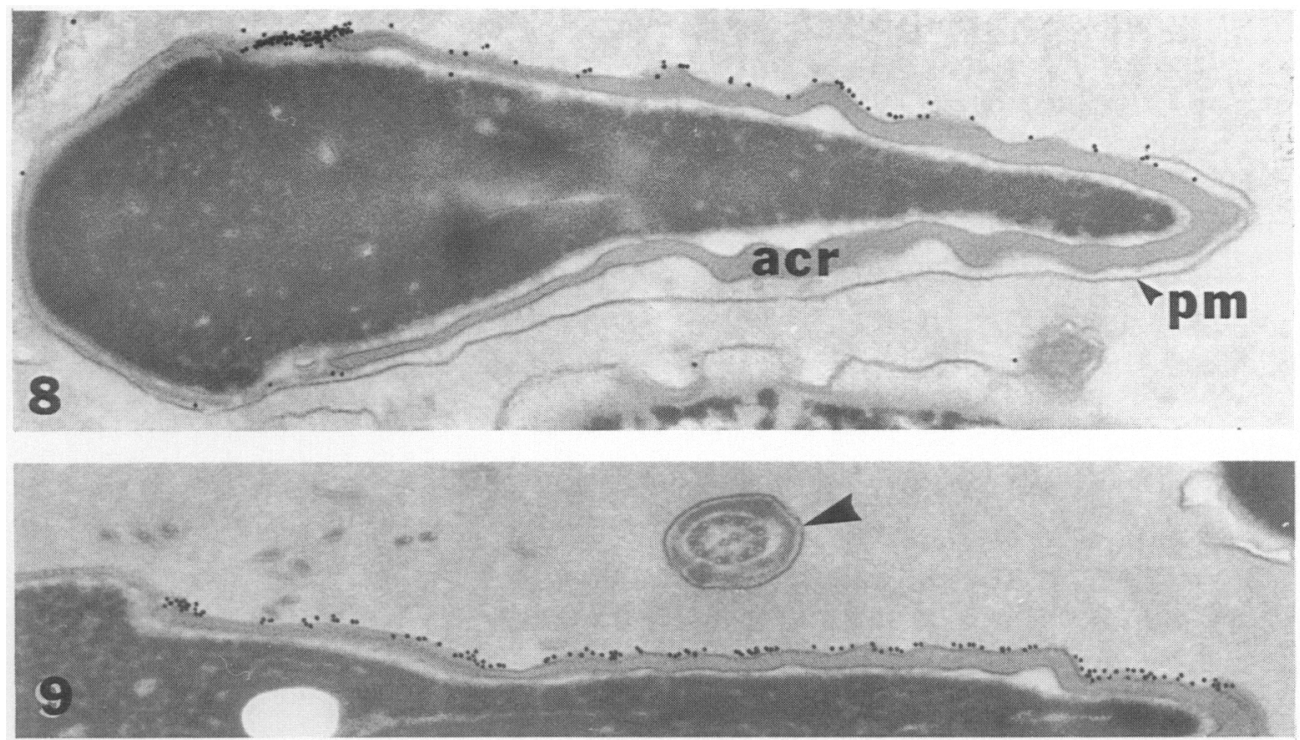

Fig. 8. Electronmicrograph of a human sperm head labelled in suspension with gold-PNA after saponin pretreatment. The gold particles are located on the outer membrane of the acrosome (acr) on the upper half of the head, but the residual plasma membrane (pm) on the lower half shows no gold particles and neither does the underlying outer acrosomal membrane. $\times 33000$.

Fig. 9. Electronmicrograph of a human sperm head and cross-section of the principal piece of the tail after labelling in suspension with gold-PNA after saponin pretreatment. The gold particles are located on the outer acrosomal membrane only. The intact plasma membrane of the tail (arrow head) shows no gold particles. $\times 33000$.

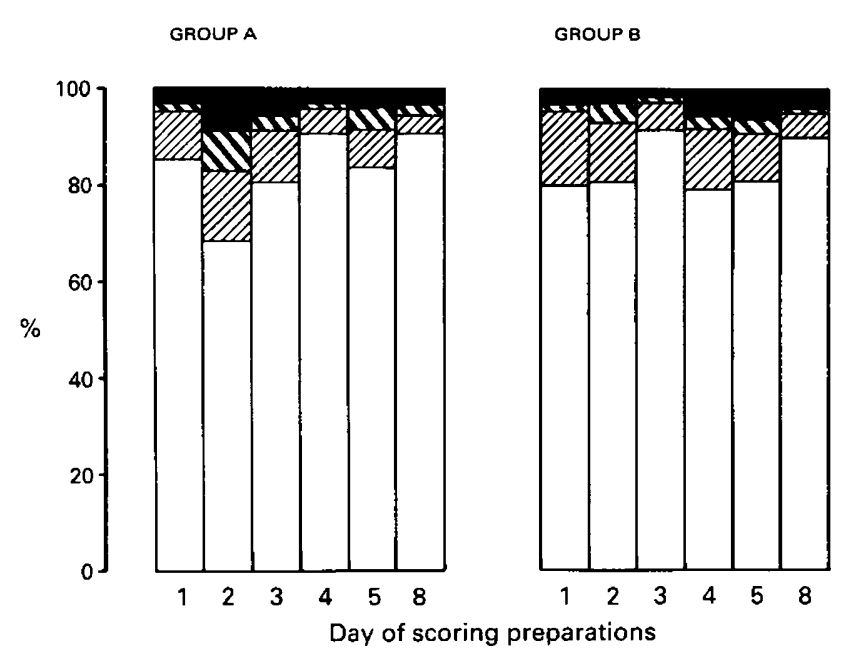

Fluorescence:

Fig. 10. Percentages of human spermatozoa showing the various patterns of FITC-PNA labelling (see Fig. 1) after storage of air-dried preparations for up to 8 days in the dark. Group A samples were stored unlabelled and unfixed, while Group B samples were labelled with the lectin and fixed with formaldehyde before storage. 
When acrosome reactions were artificially induced using the calcium ionophore A23187, there were significant increases in the proportions of spermatozoa showing both patchy acrosomal fluorescence $(P<0.05)$ and fluorescence of the equatorial segment only $(P<0.001)$. Concomitantly there was a significant $(P<0.05)$ reduction in the occurrence of bright whole acrosome fluorescence (Fig. 7).

\section{Ultrastructural localization of $P N A$ labelling}

From Figs 8 and 9 it is clear that PNA bound specifically the outer acrosomal membrane of the spermatozoa. Colloidal gold particles did not attach to the plasma membrane of either the head or tail regions of mature spermatozoa.

\section{Stability of PNA labelling during storage}

From the results of Exp. 6 it can be seen that the pattern of PNA binding to human spermatozoa was reasonably constant even after periods of up to 8 days. Preparations stored after PNAlabelling and formaldehyde fixation demonstrated the more stable results (Fig. 10).

\section{Discussion}

This series of experiments on the identification and characterization of PNA binding to human spermatozoa clearly indicates a potential role for this lectin as a marker of the human sperm acrosome and the acrosome reaction.

Since there was minimal labelling of intact spermatozoa in suspension, but extensive labelling of spermatozoa after air-drying or after disruption or removal of the plasma membrane, PNA evidently binds to intracellular and not surface determinants. Ultrastructural localization using gold-PNA demonstrated that the lectin bound to the outer acrosomal membrane.

Nonidet P-40 is an octyl phenol ethylene oxide condensate chemically closely related to Triton $\mathrm{X}-100$ which is known to remove the plasma membrane and outer acrosome membrane (primarily of the anterior cap region) of the human spermatozoon (Mortimer, 1981). Consequently, it may be concluded that the pattern of fluorescence seen after NP-40 treatment reflects PNA binding to the more resistant equatorial segment region of the acrosome after solubilization of the outer acrosomal membrane in the anterior cap region (with the concomitant loss of the acrosomal contents from that region).

The above findings do not confirm fully those of Kallajoki et al. (1985), who reported that the PNA binding after NP-40 treatment was similar to that of untreated spermatozoa. We have no explanation for this discrepancy. The study of Lee \& Damjanov (1985), which concluded that PNA reacted with the acrosomal cap region of the plasma membrane of ejaculated human spermatozoa, utilized air-dried and acetone-fixed sperm preparations exclusively. Since both procedures would result in disruption of the plasma membrane and exposure of intracellular structures, these authors' conclusion that PNA reacts with a surface glycoconjugate is untenable from their own data and must be discounted in light of the present findings. Jones et al. (1983) warned that assignment of a molecule to a regional domain on the sperm surface using fluorescence microscopy should be validated by a demonstration that it is actually located on the cell surface.

PNA has a sugar specificity very similar to that of RCA-60, the lectin used by Talbot \& Chacon $(1980,1981)$ as a marker for the human sperm acrosome. Indeed, when PNA labelling was investigated before and after a $20 \mathrm{~h}$ 'capacitation' incubation (Exp. 4) there were significant changes in the proportions of spermatozoa showing fluorescence over the entire acrosomal region, the equatorial segment only, and those showing no fluorescence. These changes may well be related to the occurrence of spontaneous acrosome reactions in these sperm populations. When acrosome reactions were artificially induced by A23187 (Exp. 5; Fig. 7) the changes in patterns of fluorescence 
were in complete accordance with expected modifications of the outer acrosomal membrane (Jamil \& White, 1981).

Lastly, the structures binding the PNA can be preserved by air-drying for at least 8 days, especially if the labelling is performed immediately after air-drying and the preparation subsequently fixed with paraformaldehyde. Provided these preparations are stored in the dark to prevent degradation of the FITC they may be scored when convenient during the days after an experiment. This is an important aspect as it allows randomization and coding of preparations so that the slides for an experiment may be scored blind to eliminate observer bias.

In conclusion, FITC-PNA is a marker for the acrosome region of human spermatozoa which may be evaluated readily by fluorescence microscopy. Cross et al. (1986) have reported the use of Pisum sativum agglutinin (PSA) from the edible pea as a marker of the human sperm acrosome. They found that PSA-labelling, which was inhibited by $\alpha$-methyl mannoside and not by $\beta$-Dgalactose, was localized within the acrosomal contents. Clearly PSA and PNA bind to different determinants within the human spermatozoon, with PSA binding to acrosome-reacted spermatozoa and PNA binding to spermatozoa which still have an outer acrosomal membrane present. Detailed evaluation of lectin-labelling for monitoring the acrosome reaction of human spermatozoa is justified, and it may replace the highly toxic RCA-60 procedure (Talbot \& Chacon, 1980, 1981). It also represents a more practicable method for routine application than the use of monoclonal antibodies (Kallajoki \& Suominen, 1984; Wolf et al., 1985; Cross et al., 1986), although the latter approach does have the possible advantage of greater molecular specificity.

Financial support for these studies was provided by The Alberta Heritage Foundation for Medical Research and the Nat Christie Foundation. We thank Miss Louise Lalonde for electronmicroscopy preparation and Mr Roland Haselden for printing the light micrographs.

\section{References}

Cross, N.L., Morales, P., Overstreet, J.W. \& Hanson, F.W. (1986) Two simple methods for detecting acrosomereacted human sperm. Gamete Res. 15, 213-226.

Dravland, J.E. \& Mortimer, D. (1985) A simple discontinuous Percoll gradient procedure for washing human spermatozoa. IRCS Med. Sci. 13, 16-17.

Glauert, A.M. (1975) Fixation, Dehydration and Embedding of Biological Specimens. North-Holland, Amsterdam.

Jamil, K. \& White, I.G. (1981) Induction of acrosomal reaction in sperm with ionophore A23187 and calcium. Archs Androl. 7, 283-292.

Jones, R., Brown, C.R., Cran, D.G. \& Gaunt, S.J. (1983) Surface and internal antigens of rat spermatozoa distinguished using monoclonal antibodies. Gamete Res. 8, 255-265.

Kallajoki, M. \& Suominen, J. (1984) An acrosomal antigen of human spermatozoa and spermatogenic cells characterized with a monoclonal antibody. Int. J. Androl. 7, 283-296.

Kallajoki, M., Malmi, R., Virtanen, I. \& Suominen, J. (1985) Glycoconjugates of human sperm surface. A study with fluorescent lectin conjugates and Lens culinaris agglutinin affinity chromatography. Cell Biol. Int. Rep. 9, 151-164.

Koehler, J.K. (1981) Lectins as probes of the spermatozoon surface. Archs Androl. 6, 197-217.

Lee, M.-C. \& Damjanov, I. (1985) Lectin binding sites on human sperm and spermatogenic cells. Anat. Rec. 212, 282-287,
Mortimer, D. (1981) The assessment of human sperm morphology in surface replica preparations for transmission electron microscopy. Gamete Res. 4, $113-119$.

Mortimer, D. (1985a) The male factor in infertility: Part 1: semen analysis. In Current Problems in Obstetrics, Gynecology and Fertility, Vol. VIII, No. 7, pp. 1-87. Year Book Medical Publishers, Chicago.

Mortimer, D. (1985b) The male factor in infertility: Part 2: sperm function testing. In Current Problems in Obstetrics, Gynecology and Fertility, Vol. VIII, No. 8, pp. 1-75. Year Book Medical Publishers, Chicago.

Mortimer, D. (1986) Comparison of the fertilizing ability of human spermatozoa preincubated in calcium- and strontium-containing media. J. exp. Zool. 237, 21-24.

Stevens, S.M.B. \& Boadle, R.A. (1987) Investigation of male infertility: abnormal spermatozoa. In Diagnostic Ultrastructure of Non-neoplastic Diseases (in press). Eds D. W. Henderson \& J. M. Papadimitriou. Churchill Livingstone, London.

Talbot, P. \& Chacon, R. (1980) A new procedure for rapidly scoring acrosome reactions of human sperm. Gamete Res. 3, 211-216.

Talbot, P. \& Chacon, R.S. (1981) Observations on the acrosome reactions of human sperm in vitro. Am. J. Primatol. 1, 211-219.

Wolf, D.P., Boldt, J., Byrd, W. \& Bechtol, K.B. (1985) Acrosomal status evaluation in human ejaculated sperm with monoclonal antibodies. Biol. Reprod. 32, 1157-1162.

Received 16 January 1987 